\title{
Prolonging Dual Antiplatelet Therapy Improves Long-term Prognosis in Diabetic Patients Undergoing Complex Percutaneous Coronary Intervention with Drug-eluting Stents
}

\author{
Xu Jingjing \\ Fuwai Hospital \\ Jia Sida \\ Fuwai Hospital \\ Zhu Pei \\ Fuwai Hospital \\ Song Ying \\ Fuwai Hospital \\ Yuan Deshan
}

Fuwai Hospital

Zhao Xueyan

Fuwai Hospital

Yao Yi

Fuwai Hospital

Jiang Lin

Fuwai Hospital

\section{Li Jianxin}

National Clinical Research Center for Cardiovascular Diseases $囚$, Chinese Academy of Medical Sciences and Peking Union Medical College

Zhang Yin

Fuwai Hospital

Song Lei

Fuwai Hospital

Gao Lijian

Fuwai Hospital

Qiao Shubin

Fuwai Hospital

Yang Yuejin

Fuwai Hospital

Xu Bo 
Fuwai Hospital

\section{Gao Runlin}

Fuwai Hospital

\section{Han Yaling}

The General Hospital of Northern Theater Command

\section{Yuan Jinqing ( $\nabla$ dr_jinqingyuan@sina.com)}

Fuwai Hospital

\section{Research Article}

Keywords: Dual antiplatelet therapy, Diabetes Mellitus, Complex coronary artery disease, Percutaneous coronary intervention, Prognosis

Posted Date: February 21st, 2022

DOI: https://doi.org/10.21203/rs.3.rs-1367685/v1

License: (c) (1) This work is licensed under a Creative Commons Attribution 4.0 International License. Read Full License 


\section{Abstract}

Background The optimal dual antiplatelet therapy (DAPT) duration after percutaneous coronary intervention (PCl) remains controversial, especially for patients at high risk of ischemic events. Diabetes mellitus (DM) and complex coronary interventions have been considered as high risk factors for ischemic events. We aim to investigate if prolonging DAPT duration in diabetic patients after drug-eluting stent (DES) implantation can reduce long-term adverse events.

Methods This study is an observational cohort study carried out in the largest cardiovascular center in China. 10,724 patients who underwent PCI from January, 2013 to December, 2013 were consecutively enrolled. The inclusion criteria included: documented history of DM; underwent DES implantation; free of adverse events within 1 year after operation; and had at least one high thrombotic risk technical aspects. These patients were divided into three groups according to DAPT duration: Standard group (11 $\leq$ DAPT $\leq 13$ months) and prolonged group (13< DAPT $\leq 24$ months; DAPT $>24$ months). Baseline characteristics and 5-year clinical outcomes were collected and analyzed.

Results A total of 2,403 diabetic patients with complex PCl were included in the current analysis (Standard DAPT $n=689$; Prolonged DAPT $n=1714$ ). Baseline and angiographic characteristics, and complexity of $\mathrm{PCl}$ were comparable among patients with different DAPT durations. Patients in $13<$ DAPT $\leq 24$ months group had lowest incidence of MACCE, all cause death and cardiac death. After adjustment of confounding factors, the 13-24 months DAPT duration was related with lower risk of MACCE (HR: $0.544,95 \% \mathrm{Cl}: 0.373-0.795)$ and all cause death (HR: $0.605,95 \% \mathrm{Cl}: 0.387-0.944)$. DAPT duration $>24$ months was related with lower risk of all-cause death (HR: $0.681,95 \% \mathrm{Cl}: 0.493-0.942$ ) and cardiac death (HR: $0.620,95 \% \mathrm{Cl}$ : 0.403-0.952). Prolonging DAPT duration did not increase the risk of major bleeding (13<DAPT $\leq 24$ months: HR: 1.356, 95\%Cl: 0.766-2.401; DAPT >24 months: HR: 0.967, 95\%Cl: 0.682-1.371).

Conclusions For diabetic patients undergoing complex PCl, prolonging DAPT duration might improve long-term prognosis by reducing the risk of adverse ischemia events and without increasing the risk of bleeding.

\section{Background}

Although dual antiplatelet therapy (DAPT) is already routinely used as secondary prevention for patients undergoing percutaneous coronary intervention $(\mathrm{PCl})$, the optimal duration of DAPT continues to be debated ${ }^{[1]}$. Diabetes mellitus (DM) has long been considered as a high-risk factor for ischemic events, patients of coronary artery disease complicated with DM showed poor prognosis ${ }^{[2]}$. Complex coronary artery disease, such as multi-vessel disease, chronic total occlusions and multi-stent implantation, are also deemed as high thrombotic risk factors. Since prolonging DAPT has been proved to reduce the incidence of ischemic events ${ }^{[3]}$, it might benefit diabetic patients undergoing complex $\mathrm{PCl}$ even more. 
However, the benefit is offset by a potential increase in risk of bleeding. Therefore, it suggested that DAPT duration should be tailored according to the risk of ischemia and bleeding in current guidelines ${ }^{[4 \sim 6]}$.

A previous study from our center reported that compared with standard DAPT duration (1 year), prolonged DAPT to more than 1 year could reduce the incidence of all-cause death, cardiac death and stent thrombosis after 2-year follow-up in patients who underwent $\mathrm{PCl}$ with drug-eluting stents (DES), without increasing the risk of bleeding ${ }^{[7]}$. However, for patient complicated with DM undergoing complex $\mathrm{PCl}$, it is not clear that whether prolonging DAPT will continue to be beneficial beyond 2 years. Therefore, we further investigated 5-year clinical outcomes to evaluate the exact impact of different DAPT durations on long-term prognosis.

\section{Patients And Methods}

\section{Study population}

A total of 10,724 patients underwent PCl at Fu Wai Hospital, National Center for Cardiovascular Diseases (Beijing, China) from January to December 2013. The exclusion criteria includes: patients received only bare metal stents $(n=64)$, treated with balloon angioplasty alone $(n=237)$, had adverse cardiovascular and cerebrovascular events or bleeding events within 1 year after $\mathrm{PCI}(\mathrm{n}=612)$, DAPT duration < 1 year $(n=157)$, and single antiplatelet therapy $(n=120)$. Finally, 2,403 patients with confirmed diagnosis of diabetes mellitus and at least one high thrombotic risk technical aspects were enrolled. Patients were divided into three groups according to DAPT duration: standard group (11 $\leq$ DAPT $\leq 13$ months, $n=689$ ) and prolonged group (13< DAPT $\leq 24$ months, $n=1133$, DAPT $>24$ months, $n=581$ ). (Fig.1).

\section{Procedures and medications}

$\mathrm{PCl}$ strategy and stent type were at the treating physician's discretion. Before the procedure, patients not taking long-term aspirin and clopidogrel received a loading dose of $300 \mathrm{mg}$ aspirin and $300 \mathrm{mg}$ or 600 $\mathrm{mg}$ clopidogrel orally. During the procedure, unfractionated heparin $(100 \mathrm{U} / \mathrm{kg})$ was administered to all patients, with glycoprotein IIb/IIla inhibitors in accordance with the operator's judgment. In 2013, other P2Y12 inhibitors such as prasugrel and ticagrelor were not routinely used in our center, only 16 patients $(0.16 \%)$ received ticagrelor (loading dose: $180 \mathrm{mg}$ ) during that time.

\section{Patient follow-up}

All patients were evaluated by clinic visits or by phone at 1, 3, 6 months and 1,2, 5 years after the procedure. Patients were advised to return for coronary angiography if clinically indicated by symptoms or if showing signs of myocardial ischemia.

\section{Definitions}

The duration of DAPT was defined as the length between the date of PCl and DAPT cessation. Patients were divided into two groups according to different DAPT duration: standard DAPT 
(11months $\leq$ DAPT $\leq 13$ months, $n=689$ ) and prolonged DAPT group (DAPT duration $>13$ months, $\mathrm{n}=1714$ ). Prolonged DAPT group was further divided into two subgroups: 13 months $<$ DAPT $\leq 24 \mathrm{months}$, $\mathrm{n}=1133$ and DAPT $>24$ months, $\mathrm{n}=581$. (Fig.1). Myocardial Infarction (MI) was defined according to the third universal definition of myocardial infarction ${ }^{[8]}$. Bleeding was defined according to the definition of the Bleeding Academic Research Consortium ${ }^{[9]}$. New-generation DES was defined as second-generation DES and biodegradable polymer DES.

The definition of diagnostic criteria for diabetes mellitus refer to the guideline of the prevention and treatment of type 2 diabetes mellitus in China (2020 Edition): definite history of diabetes mellitus or symptoms of diabetes mellitus complicated with fasting blood glucose $\geq 7.0 \mathrm{mmol} / \mathrm{L}$ or HbAlc $\geq 6.5 \%$ [10]. The high thrombotic risk technical aspects refers to the risk criteria for extended treatment with a second antithrombotic agent from 2020 ESC Guidelines for the management of acute coronary syndromes in patients presenting without persistent ST-segment elevation, including at least 3 stents implanted, at least 3 lesions treated, total stent length $>60 \mathrm{~mm}$, history of complex revascularization (left main, bifurcation stenting with $\geq 2$ stents implanted, chronic total occlusion) [11].

\section{Endpoints}

The primary endpoint was major adverse cardiovascular and cerebrovascular events (MACCE), a composite of all-cause death, MI and stroke at 5-year follow up. The secondary endpoints were the individual components of MACCE and bleeding. All endpoint events were adjudicated by two independent cardiologists, and disagreement was resolved by consensus.

\section{Statistical analysis}

Continuous variables were compared by one-way ANOVA test, as appropriate. Categorical variables were compared by using Bonferroni method in Z-test between columns, and the overall p value was calculated by Pearson's $\chi^{2}$ test. Continuous variables were presented as mean \pm standard deviation and categorical variables as number with frequency. Survival curves were calculated using Kaplan-Meier estimates and compared with the log-rank test regarding endpoints. We took the standard DAPT as reference and justified variables of age, gender, BMI, hypertension, hyperlipidemia, previous PCI and SNYTAX score before PCI for multivariable Cox regression models. Results of COX regression analysis were expressed as Hazard Ratio (HR) and 95\% confidence interval (Cl). Subgroup analyses were performed using factors of high ischemia and high bleeding risk (age, gender, history of smoking, previous $\mathrm{PCl}$ or previous $\mathrm{MI}$, ACS, cardiac function and renal function) to analyze interactions between DAPT duration and the selected factors for the outcomes.

Two-tailed P values of $<0.05$ were considered to be statistically significant. All analyses were conducted with SPSS version 20.0 (IBM, Armonk, NY, USA).

\section{Result}




\section{Baseline Characteristics}

Standard DAPT accounted for 28.7\% (689/2403) and prolonged DAPT for 71.3\% (1714/2403) respectively. Patients' baseline characteristics were shown in Table 1. The basic and angiographic characteristics were similar among the three groups of different DAPT durations, except for a lower incidence of previous CABG in $13<$ DAPT $\leq 24$ months group, a higher level of fasting blood glucose in DAPT >24 months group, and a higher incidence of unstable angina in $11 \leq$ DAPT $\leq 13$ months group.

Table 1 Baseline characteristics among three DAPT duration groups 


\begin{tabular}{|c|c|c|c|c|c|}
\hline & \multirow{3}{*}{$\begin{array}{l}\text { Standard } \\
\text { DAPT } \\
11-13 \text { mos. } \\
n=689\end{array}$} & \multicolumn{4}{|c|}{ Prolonging DAPT } \\
\hline & & 13-24 mos. & $>24$ mos. & $F / \chi^{2}$ & $P$ value \\
\hline & & $n=1133$ & $n=581$ & & \\
\hline Age,y & $58.40 \pm 10.28$ & $57.82 \pm 10.07$ & $58.67 \pm 10.13$ & 1.567 & 0.209 \\
\hline Female, \% & $150(21.8)$ & $275(24.3)$ & $142(24.4)$ & 1.790 & 0.409 \\
\hline $\mathrm{BMI}, \mathrm{kg} / \mathrm{m} 2$ & $25.95 \pm 3.12$ & $25.99 \pm 3.10$ & $26.11 \pm 3.02$ & 0.439 & 0.645 \\
\hline \multicolumn{6}{|l|}{ Risk factors } \\
\hline Hypertension, \% & $446(64.7)$ & $754(64.5)$ & $370(63.7)$ & 1.548 & 0.461 \\
\hline Hyperlipidemia, \% & $446(64.7)$ & $784(69.2)$ & $397(68.3)$ & 4.043 & 0.132 \\
\hline Family history of CAD , \% & $150(21.8)$ & $259(22.9)$ & $145(25.0)$ & 1.850 & 0.397 \\
\hline Smokeing, \% & $386(56.0)$ & $623(55.0)$ & $323(55.6)$ & 0.647 & 0.958 \\
\hline Previous MI, \% & $120(17.4)$ & $208(18.4)$ & $108(18.6)$ & 0.358 & 0.836 \\
\hline Previous PCl, \% & $151(21.9)$ & $254(22.4)$ & $158(27.2)_{\mathrm{ab}}$ & 6.116 & $0.047^{\star}$ \\
\hline Previous CABG, \% & $44(6.4)$ & $26(2.3)_{\mathrm{ac}}$ & $33(5.7)$ & 21.108 & $<0.001^{*}$ \\
\hline Previous stroke, \% & $82(11.9)$ & $123(10.9)$ & $67(11.5)$ & 0.501 & 0.778 \\
\hline $\begin{array}{l}\text { Peripheral vascular } \\
\text { disease, \% }\end{array}$ & $20(2.9)$ & $23(2.0)$ & $21(3.6)$ & 3.933 & 0.140 \\
\hline \multicolumn{6}{|l|}{ Examination } \\
\hline $\begin{array}{l}\text { Fasting blood glucose, } \\
\mathrm{mmol} / \mathrm{L}\end{array}$ & $6.77 \pm 2.49$ & $6.62 \pm 2.36$ & $6.96 \pm 2.57 \mathrm{ab}$ & 3.385 & $0.022^{\star}$ \\
\hline HbAlc, \% & $6.94 \pm 1.27$ & $6.97 \pm 1.40$ & $6.94 \pm 1.35$ & 0.146 & 0.864 \\
\hline HGB before PCl, g/L & $141.21 \pm 15.98$ & $140.23 \pm 16.03$ & $141.64 \pm 15.14$ & 1.757 & 0.173 \\
\hline PLT before $\mathrm{PCl}, * 10^{9} / \mathrm{L}$ & $205.50 \pm 56.60$ & $208.54 \pm 53.66$ & $204.52 \pm 52.83$ & 1.279 & 0.279 \\
\hline WBC, ${ }^{*} 10^{9} / \mathrm{L}$ & $7.02 \pm 1.96$ & $6.99 \pm 1.90$ & $6.95 \pm 1.95$ & 0.217 & 0.805 \\
\hline $\mathrm{TC}, \mathrm{mmol} / \mathrm{L}$ & $4.30 \pm 1.14$ & $4.26 \pm 1.13$ & $4.20 \pm 1.04$ & 1.253 & 0.286 \\
\hline $\mathrm{TG}, \mathrm{mmol} / \mathrm{L}$ & $1.88 \pm 1.08$ & $1.87 \pm 1.30$ & $1.78 \pm 1.06$ & 1.181 & 0.307 \\
\hline LDL-C, mmol/L & $2.56 \pm 0.94$ & $2.53 \pm 0.94$ & $2.50 \pm 0.88$ & 0.514 & 0.598 \\
\hline HDL-C, mmol/L & $1.04 \pm 0.29$ & $1.03 \pm 0.29$ & $1.03 \pm 0.28$ & 0.101 & 0.904 \\
\hline $\mathrm{UA}, \mu \mathrm{mol} / \mathrm{L}$ & $346.88 \pm 83.94$ & $337.70 \pm 87.27$ & $342.92 \pm 90.64$ & 2.446 & 0.087 \\
\hline
\end{tabular}




\begin{tabular}{llllll} 
eGFR, $\mathrm{ml} / \mathrm{min}$ & $92.58 \pm 14.36_{\mathrm{bc}}$ & $90.63 \pm 15.51$ & $90.73 \pm 14.96$ & 3.986 & $0.019 *$ \\
\hline LVEF, \% & $61.87 \pm 7.84$ & $62.04 \pm 7.59$ & $61.34 \pm 7.76$ & 1.562 & 0.210
\end{tabular}

\section{Clinical and Angiographic characteristics}

\begin{tabular}{llllll}
\hline Pre-Syntax Score & $15.27 \pm 8.49$ & $15.29 \pm 8.17$ & $15.34 \pm 8.12$ & 0.010 & 0.990 \\
\hline No. of target lesion & $1.78 \pm 0.81$ & $1.78 \pm 0.82$ & $1.84 \pm 0.83$ & 1.278 & 0.279 \\
\hline No. of stents per patient & $2.54 \pm 1.11$ & $2.67 \pm 1.17$ & $2.65 \pm 1.26$ & 2.913 & 0.055 \\
\hline New generation DES, \% & $585(84.9)$ & $962(84.9)$ & $492(84.7)$ & 4.322 & 0.364 \\
\hline ACS, \% & $445(64.6)_{\mathrm{b}}$ & $640(56.5)$ & $338(58.2)$ & 11.982 & $0.003^{\star}$ \\
\hline AMI, \% & $109(15.8)$ & $196(17.3)$ & $93(15.8)$ & 0.941 & 0.625 \\
\hline UA, \% & $336(48.8)_{\mathrm{b}}$ & $444(39.2)$ & $245(42.2)$ & 10.552 & $0.005^{\star}$
\end{tabular}

Standard DAPT: 11months $\leq$ DAPT duration $\leq 13$ months, Prolonged DAPT: DAPT duration $>13$ months

$\mathrm{BMI}=$ body mass index, $\mathrm{CABG}=$ coronary artery bypass grafting, $\mathrm{CAD}=$ coronary artery disease, $\mathrm{DAPT}=$ dual antiplatelet therapy, GFR=glomerular filtration rate, $\mathrm{HbAlc}=$ glycosylated hemoglobin, $\mathrm{HDL}-\mathrm{C}=\mathrm{High}$ density lipoprotein cholesterol, LDL-C=Low density lipoprotein cholesterol, LVEF=left ventricular ejection fraction, $\mathrm{MI}=$ myocardial infarction, $\mathrm{PCl}=$ percutaneous coronary intervention, $\mathrm{PLT}=$ platelet, $\mathrm{HGB}=$ hemoglobin, $\mathrm{AMI}=$ Acute myocardial infarction, $\mathrm{TC}=$ total cholesterol, $\mathrm{TG}=$ triglyceride

*: $p<0.05$

a means significant difference with group 11 months $\leq$ DAPT $\leq 13$ months, $b$ means significant difference with group 13 months $<$ DAPT $\leq 24$ months, $c$ means significant difference with group DAPT $>24$ months (in 0.05 level).

\section{Five-year Outcomes}

During 5-year follow-up, the incidences of MACCE, all cause death and cardiac death in prolonged DAPT groups were significantly lower than standard DAPT group, with the group of $13<$ DAPT $\leq 24$ months being the lowest $(11 \leq$ DAPT $\leq 13$ months vs. $13<$ DAPT $\leq 24$ months vs. DAPT $>24$ months: MACCE $8.1 \%$ vs. $4.6 \%$ vs. $6.0 \%, p=0.008$, all cause death $4.6 \%$ vs. $1.9 \%$ vs. $2.2 \%$, $p=0.002$, cardiac death: $3.0 \%$ vs. $1.0 \%$ vs. $1.2 \%, p=0.002$, respectively). The incidence of stroke and bleeding had no differences among the three groups (Table 2). A Kaplan-Meier estimate showed similar trend (Fig. 3).

Table 2. 5-year outcomes among different DAPT duration 


\begin{tabular}{|c|c|c|c|c|c|}
\hline & \multirow{3}{*}{$\begin{array}{l}\text { Standard DAPT } \\
\text { 11-13 mos. } \\
n=689\end{array}$} & \multicolumn{4}{|c|}{ Prolonged DAPT } \\
\hline & & & & $x^{2}$ & $P$ value \\
\hline & & $n=1133$ & $\mathrm{n}=581$ & & \\
\hline MACCE & $56(8.1)_{b}$ & $52(4.6)$ & $35(6.0)$ & 9.591 & $0.008 *$ \\
\hline All cause death & $32(4.6)_{b}$ & $22(1.9)$ & $13(2.2)$ & 12.404 & $0.002 *$ \\
\hline Cardiac death & $21(3.0)_{b}$ & $11(1.0)$ & $7(1.2)$ & 12.416 & $0.002 *$ \\
\hline Myocardial infarction & $30(4.4)$ & $33(2.9)$ & $30(5.2)$ & 5.838 & 0.054 \\
\hline Stroke & $26(3.8)$ & $28(2.5)$ & 19 (3.3) & 2.607 & 0.272 \\
\hline Major bleeding & $17(2.5)$ & $39(3.4)$ & $15(2.6)$ & 1.792 & 0.408 \\
\hline
\end{tabular}

Standard DAPT: 11months $\leq$ DAPT duration $\leq 13$ months, Prolonged DAPT: DAPT duration $>13$ months MACCE = major adverse cardiac and cerebrovascular events, Mos: months

$\star: p<0.05$

a means significant difference with group 11 months $\leq$ DAPT $\leq 13$ months, $b$ means significant difference with group 13 months $<$ DAPT $\leq 24$ months, c means significant difference with group DAPT $>24$ months (in 0.05 level).

\section{High thrombotic risk technical aspects}

The high thrombotic risk technical aspects of patients with different DAPT duration are shown in figure 2 . There was no significant difference of complex coronary intervention technique among three DAPT duration groups.

\section{Multivariable analysis}

COX multivariable regression analysis showed that compared with standard DAPT duration, prolonged DAPT to 13-24 months significantly reduced the risk of MACCE (HR: $0.544,95 \% \mathrm{Cl}: 0.373-0.795)$ and allcause death (HR: $0.605,95 \% \mathrm{Cl}: 0.387-0.944)$. DAPT duration $>24$ months significantly reduced the risk of all cause death (HR: $0.681,95 \% \mathrm{Cl}: 0.493-0.942)$ and cardiac death (HR: $0.620,95 \% \mathrm{Cl}: 0.403-0.952)$. (Table 3).

Table 3. COX regression analysis of prolonging DAPT for long-term prognosis 


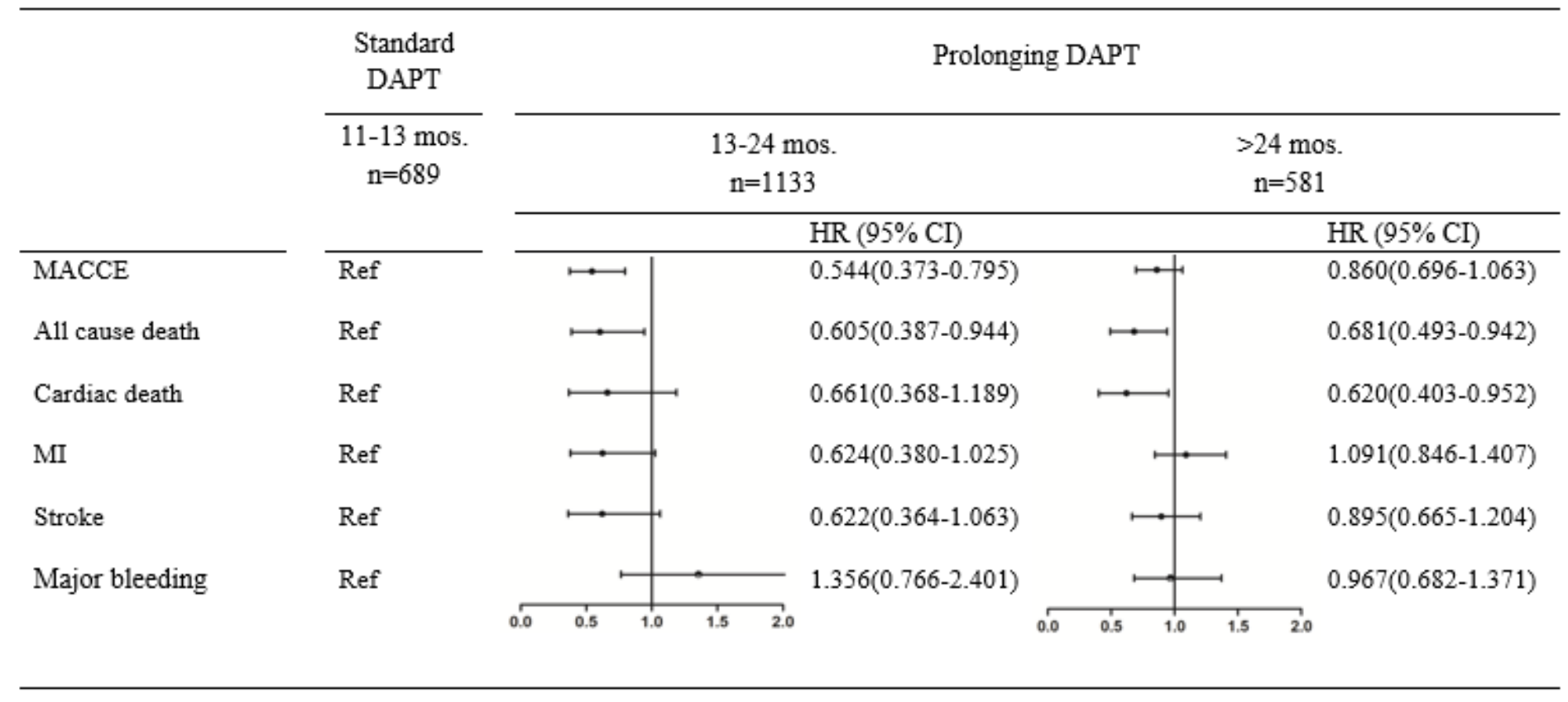

Adjusted Variables: age, gender, hypertension, hyperlipidemia, previous PCI, SNYTAX score before PCI MACCE = major adverse cardiac and cerebrovascular events, $\mathrm{Ml}=$ myocardial infarction, Mos: months

\section{Subgroup analysis}

We performed subgroup analysis on all-cause death and MACCE between standard DAPT and 13-24 months DAPT to evaluate patients with risk enhancers of ischemia or bleeding. Variables for subgroup analysis included age, gender, smoking history, previous $\mathrm{PCl}$ or previous MI history, ACS, cardiac dysfunction (LVEF $<40 \%$ ) and renal dysfunction (eGFR $<60 \mathrm{ml} / \mathrm{min} / 1.73 \mathrm{~m} 2)$. DAPT between $13-24$ months could reduce the risk of all cause death and MACCE in the subgroups of age $\geq 65$ years and $<65$ years old, male patients, patients with smoking history, patients with and without previous $\mathrm{PCl}$ or previous $\mathrm{MI}$ history, ACS patients, patients with and without cardiac dysfunction and patients without renal dysfunction. As shown in Table 4.

Table 4. Subgroup analyses on all-cause death and MACCE between standard DAPT and 13-24 months DAPT 


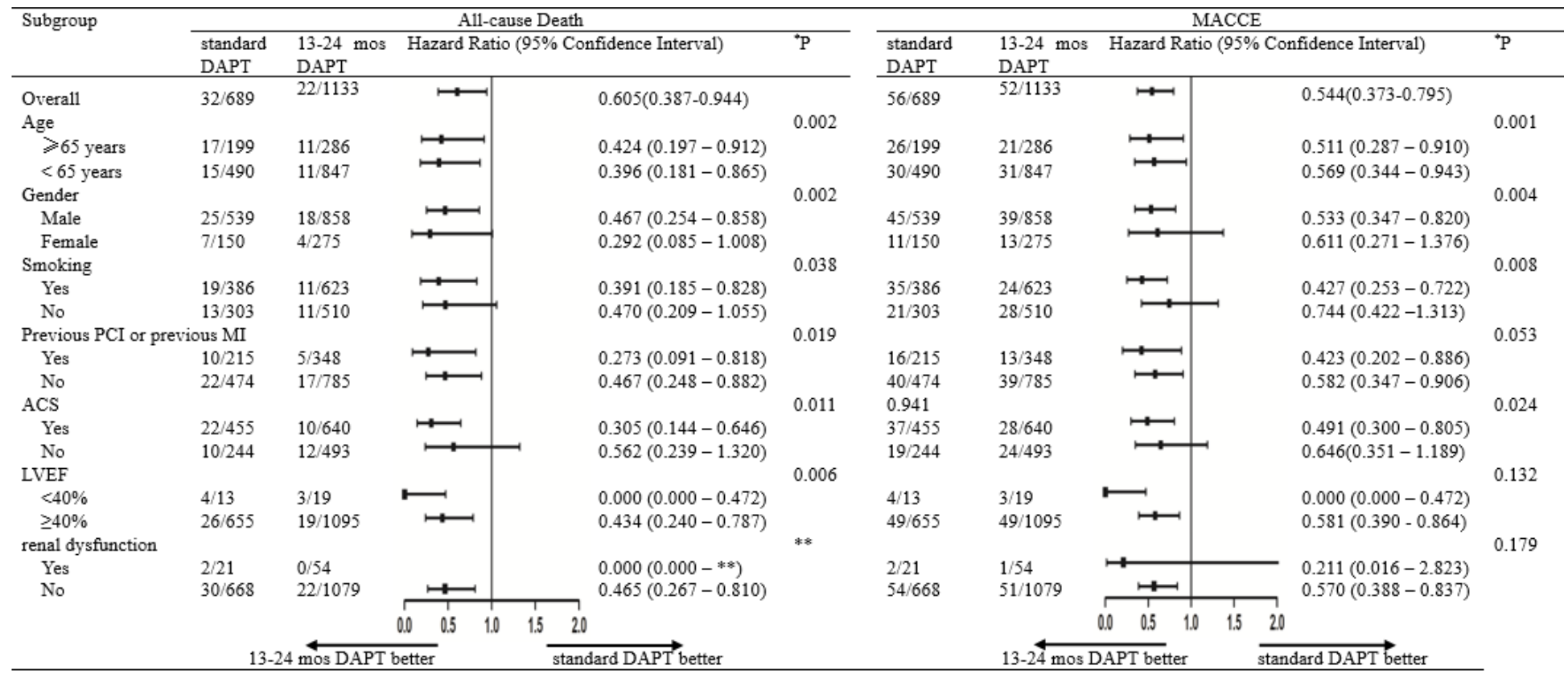

${ }^{*} \mathrm{P}$ value for interaction in each subgroup analysis

${ }^{* *}$ Unable to calculate in this subgroup due to unreliable results of renal dysfunction subgroup

Adjusted Variables: age, gender, BMI, hypertension, hyperlipidemia, previous PCI, SNYTAX score before $\mathrm{PCl}$

LVEF = Left Ventricular Ejection Fraction

\section{Discussion}

The objective of this study is to investigate appropriate DAPT duration for patients at high risk of ischemic events such as diabetic patients and patients undergoing complex $\mathrm{PCl}$. The major findings are as follows: (1) Compared with standard DAPT duration, prolonged DAPT duration in high ischemia risk patients undergoing $\mathrm{PCl}$ with DES is common in China. (2) For patients complicated with high ischemic risk, such as DM or complex coronary intervention, prolonged DAPT duration could reduce incidence of adverse cardiovascular events. (3) Prolonged DAPT does not increase the risk of bleeding. (4) Patients at high ischemic risk benefits even more when combined with risk enhancers of ischemic events.

\section{DM and complex coronary intervention increase ischemic risk}

DM is a major risk factor for coronary heart disease, which makes patients vulnerable to atherosclerosis due to the damage of vascular endothelium and vascular dysfunction caused by metabolic abnormalities of blood glucose. In addition, high platelet reactivity and hypercoagulability in patients with DM also increase the risk of thrombosis and ischemic events significantly ${ }^{[12]}$. Complex coronary interventions could also increase the risk of thrombosis. Although advancement in interventional equipments and 
techniques have largely enabled interventional cardiologists to revascularize extremely difficult coronary lesions ${ }^{[13]}$, the ischemic risk should be paid more attention.

\section{Selection of DAPT duration and guidelines recommendation}

DAPT benefits patients undergoing PCl by reducing the incidence of stent thrombosis and strengthening the secondary prevention of coronary heart disease ${ }^{[14]}$. The American and European guidelines recommended a 12-month DAPT for patients after DES implantation and adjusting DAPT duration according to the risk of ischemic and bleeding ${ }^{[15,16]}$. Guidelines recommend appropriately extending of DAPT duration if patients had complex CAD combined with high-risk factors of ischemia ${ }^{[4]}$.

In the current study, standard DAPT accounted for $28.7 \%$ and prolonged DAPT for $71.3 \%$, respectively. The high proportion of prolonging DAPT reflects cardiologists' preference to extend DAPT in patients with high ischemic risk .

\section{Benefits of prolonging DAPT duration}

The benefit of prolonging DAPT is still controversial. The TIMI 38 Coronary Stent Registry showed similar recurrent ischemic events between patients continued and discontinued DAPT ( $p=0.74$ for cardiovascular death/myocardial infarction/stroke, $p=0.72$ for definite or probable stent thrombosis) ${ }^{[18]}$. The DAPT Study indicated that continued treatment with thienopyridine could reduce the rates of stent thrombosis ( $0.4 \%$ vs. $1.4 \%, \mathrm{HR} 0.29$ [95\% Cl: 0.17 to 0.48$], \mathrm{P}<0.001)$ and major adverse cardiovascular and cerebrovascular events ( $4.3 \%$ vs. $5.9 \%$, hazard ratio, 0.71 [95\% Cl: 0.59 to 0.85$], P<0.001)^{[3]}$. However, these studies did not strictly distinguish the risk of ischemia.

In our study, prolonged DAPT was associated with a decrease of adverse cardiovascular events in 5-year follow-up in diabetic patients undergoing complex PCI. DAPT duration between 13 to 24 months group has the lowest incidences of MACCE, all cause death, and cardiac death. For patients with high ischemic risk, prolonged DAPT duration appropriately is beneficial by reducing adverse cardiovascular ischemic events. Therefore, risk stratification for patients after DES is essential in order to guide the decisionmaking of DAPT duration.

\section{Prolonging DAPT does not increase bleeding risk}

The biggest concern about prolonging DAPT is the increased risk of bleeding, especially for Chinese patients, who are considered at higher risk of bleeding ${ }^{[19]}$. In our study, no significant difference in risk of bleeding was found among the three groups, which proves the safety of prolonging DAPT.

\section{More benefits for patients combined with risk enhancers}

Subgroup analysis with risk enhancers of ischemia or bleeding in the current study showed that, prolonging DAPT to 13-24 months reduced the risk of all-cause death and MACCE for the subgroups of age $\geq 65$ years and $<65$ years old, male patients, patients with smoking history, patients with and without 
previous $\mathrm{PCl}$ or previous $\mathrm{MI}$ history, ACS patients, patients with and without cardiac dysfunction and patients without renal dysfunction. These results indicated that, for patients with high ischemic risk, appropriately prolonging of DAPT duration would bring more benefits if combined with risk enhancers. The more risk factors of ischemia combined, the more definite benefits patients would gain from prolonging DAPT duration.

\section{Limitations}

Several limitations of this study should be considered. First, being an observational study, evaluation of medication efficacy is limited. In addition, the observational study was designed non-randomized with unmeasured confounders that may preclude definitive conclusions. Second, only patients who could tolerate prolonged DAPT were enrolled, which may introduce selection bias into this analysis. Third, information on how DAPT was discontinued was not available.

\section{Conclusions}

For diabetic patients undergoing complex $\mathrm{PCl}$, appropriately prolonging DAPT duration could improve long-term prognosis by reducing the risk of adverse cardiovascular ischemia events without increasing the risk of bleeding.

\section{Declarations}

\section{Ethics approval and consent to participate}

The study protocol was approved by the ethics committee of Fuwai Hospital (Approval No. 2021-1501), and written informed consent was provided by patients before the intervention.

Consent for publication: Not applicable.

Availability of data and materials. Not applicable.

Competing interests. Not applicable.

\section{Funding}

The present study was approved by The National Key Research and Development Program of China, Grant Number: 2016YFC130130 (Subtopic: 2016YFC1301301), The National Clinical Research Center for Cardiovascular Diseases, Fuwai Hospital, Chinese Academy of Medical Sciences (Grant No. NCRC2020013), The National Natural Science Foundation of China (Grant No. 81900323), China International Exchange and Promotion Association for Medical and Healthcare and Sanofi (Hangzhou) pharmaceuticals Co., Ltd (CN174125, DIREGL08735-DAPT).

\section{Authors' contributions}


XJJ, contributed to project design and article writing, JSD, ZP, SY, YDS, contributed to data collection and arrangement, ZXY, contributed to project design, YY, JL, LJX, contributed to data sorting, patients followup and statistics, ZY, SL, GLJ, QSB, YYJ, XB, contributed to project design and operation, GRL, HYL,YJQ, project design and overall plan.

\section{Acknowledgements}

The authors are grateful to the staff in the Department of Cardiology and Catheterization Laboratory, Fu Wai Hospital for their research contributions.

\section{References}

1. Tullio Palmerini, Gregg W Stone. Optimal duration of dual antiplatelet therapy after drug-eluting stent implantation: conceptual evolution based on emerging evidence. Eur Heart J. 2016, 37:353-64.

2. Ryo Naito, Katsumi Miyauchi. Coronary Artery Disease and Type 2 Diabetes Mellitus. Int Heart J. 2017,58:475-480.

3. Mauri L, Kereiakes DJ, Yeh RW, Driscoll-Shempp P, Cutlip DE, Steg PG, et al. Twelve or 30 months of dual antiplatelet therapy after drug-eluting stents. N Engl J Med. 2014, 371:2155-2166.

4. Glenn N Levine, Eric R Bates, John A Bittl, Ralph G Brindis, Stephan D Fihn, Lee A Fleisher, et al. 2016 ACC/AHA guideline focused update on duration of dual antiplatelet therapy in patients with coronary artery disease: A report of the american college of cardiology/american heart association task force on clinical practice guidelines: An update of the 2011 ACCF/AHA/SCAI guideline for percutaneous coronary intervention, 2011 ACCF/ AHA guideline for coronary artery bypass graft surgery, 2012 ACC/AHA/ACP/AATS/ PCNA/SCAI/STS guideline for the diagnosis and management of patients with stable ischemic heart disease, 2013 ACCF/AHA guideline for the management of st-elevation myocardial infarction, 2014 AHA/ACC guideline for the management of patients with non-stelevation acute coronary syndromes, and 2014 ACC/AHA guideline on perioperative cardiovascular evaluation and management of patients undergoing noncardiac surgery. Circulation. 2016, 134: e192-194.

5. Stephan Windecker, Philippe Kolh, Fernando Alfonso, Jean-Philippe Collet, Jochen Cremer, Volkmar Falk, et al. 2014 ESC/EACTS guidelines on myocardial revascularization: the Task Force on Myocardial Revascularization of the European Society of Cardiology and the European Association for Cardio-Thoracic Surgery developed with the special contribution of the European Association of Percutaneous Cardiovascular Interventions. Eur Heart J. 2014, 35: 2541-2619.

6. Chinese Society of Cardiology (CSC), Chinese College of Cardiovascular Physicians (CCCP) / Chinese Medical Doctor Association (CMDA), Editorial board of Chinese Journal of Cardiology. 2016 guideline for percutaneous coronary intervention in China. Chin J Cardiol. 2016, 44: No.5.

7. Jingjing Xu, Ying Song, Zhan Gao, Ping Jiang, Ru Liu, Huanhuan Wang, et al. Long-term outcomes of extending dual antiplatelet therapy after drug-eluting stent implantation for acute coronary syndrome: a large single-center study. Platelets. 2020, 31: 869-876. 
8. Kristian Thygesen, Joseph S Alpert, Allan S Jaffe, Maarten L Simoons, Bernard R Chaitman, Harvey D White, et al. Third universal definition of myocardial infarction. J Am Coll Cardiol. 2012, 60:1581-98.

9. Mehran R, Rao SV, Bhatt DL, Gibson CM, Caixeta A, Eikelboom J, et al. Standardized bleeding definitions for cardiovascular clinical trials: a consensus report from the Bleeding Academic Research Consortium. Circulation. 2011, 123: 2736-2747.

10. Chinese Diabetes Society. Guideline for the prevention and treatment of type 2 diabetes mellitus in China (2020 edition). Chin J Diabetes Mellitus. 2021, 13: 315-409.

11. Jean-Philippe Collet, Holger Thiele, Emanuele Barbato, Olivier Barthélémy, Johann Bauersachs, Deepak L Bhatt, Paul Dendale, et al. 2020 ESC Guidelines for the management of acute coronary syndromes in patients presenting without persistent ST-segment elevation. Eur Heart J. 2021, 42: 1289-1367.

12. Alexandros Patsouras, Paraskevi Farmaki, Anna Garmpi, Christos Damaskos, Nikolaos Garmpis, Dimitrios Mantas, et al. Screening and Risk Assessment of Coronary Artery Disease in Patients With Type 2 Diabetes: An Updated Review. In Vivo. 2019, 33:1039-1049.

13. Gianfranco De Candia. Percutaneous coronary intervention risk scores. Minerva Cardioangiol. 2018, 66:569-575.

14. Joseph Pultar, Patricia P Wadowski, Simon Panzer, Thomas Gremmel, et al. Oral antiplatelet agents in cardiovascular disease. Vasa. 2019, 48:291-302.

15. Glenn N Levine, Eric R Bates, John A Bittl, Ralph G Brindis, Stephan D Fihn, Lee A Fleisher, et al. 2016 ACC/AHA Guideline Focused Update on Duration of Dual Antiplatelet Therapy in Patients With Coronary Artery Disease: A Report of the American College of Cardiology/American Heart Association Task Force on Clinical Practice Guidelines: An Update of the 2011 ACCF/AHA/SCAI Guideline for Percutaneous Coronary Intervention, 2011 ACCF/AHA Guideline for Coronary Artery Bypass Graft Surgery, 2012 ACC/AHA/ACP/AATS/PCNA/SCAI/STS Guideline for the Diagnosis and Management of Patients With Stable Ischemic Heart Disease, 2013 ACCF/AHA Guideline for the Management of ST-Elevation Myocardial Infarction, 2014 AHA/ACC Guideline for the Management of Patients With Non-ST-Elevation Acute Coronary Syndromes, and 2014 ACC/AHA Guideline on Perioperative Cardiovascular Evaluation and Management of Patients Undergoing Noncardiac Surgery. Circulation. 2016, 134: e123-55.

16. Marco Valgimigli, Héctor Bueno, Robert A Byrne, Jean-Philippe Collet, Francesco Costa, Anders Jeppsson, et al. 2017 ESC focused update on dual antiplatelet therapy in coronary artery disease developed in collaboration with EACTS: The Task Force for dual antiplatelet therapy in coronary artery disease of the European Society of Cardiology (ESC) and of the European Association for Cardio-Thoracic Surgery (EACTS). Eur Heart J. 2018, 39: 213-260.

17. Atherosclerosis and Coronary Heart Disease Working Group of Chinese Society of Cardiology, Interventional Cardiology Working Group of Chinese Society of Cardiology, Specialty Committee on Prevention and Treatment of Thrombosis of Chinese College of Cardiovascular Physicians, Specialty Committee on Coronary Artery Disease and Atherosclerosis of Chinese College of Cardiovascular 
Physicians, Editorial Board of Chinese Journal of Cardiology. Chinese Society of Cardiology and Chinese College of Cardiovascular Physicians Expert Consensus statement on dual antiplatelet therapy in patients with coronary artery disease. Zhonghua Xin Xue Guan Bing Za Zhi. 2021, 49:432454.

18. Bonaca MP, Murphy SA, Miller D, Herrman JP, Gottlieb S, Keltai M, et al. Patterns of long-term thienopyridine therapy and outcomes in patients with acute coronary syndrome treated with coronary stenting: Observations from the TIMI-38 Coronary Stent Registry.Clin Cardiol. 2014, 37: 293-299.

19. Kang J, Park KW, Palmerini T, Stone GW, Lee MS, Colombo A, et al. Racial Differences in Ischaemia/Bleeding Risk Trade-Off during Anti-Platelet Therapy: Individual Patient Level Landmark Meta-Analysis from Seven RCTs. Thromb Haemost. 2019, 119: 149-162.

\section{Figures}

\section{Figure 1}

Flow Chart

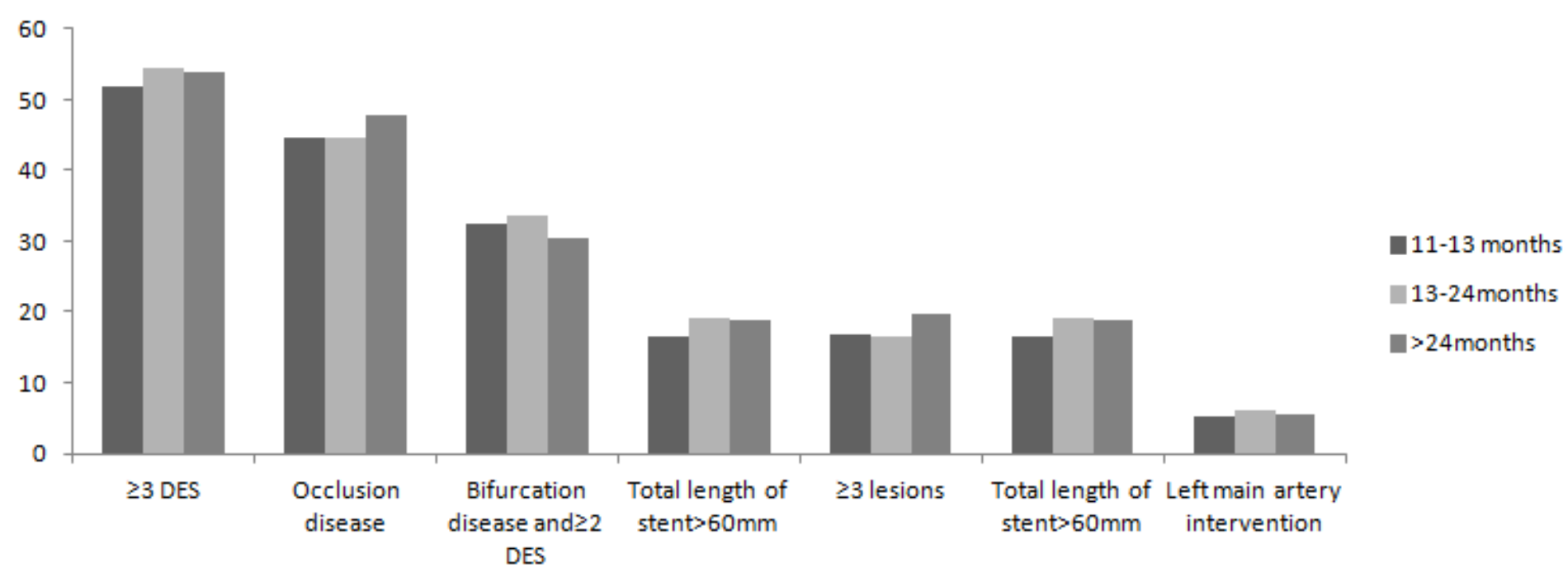

Figure 2

High thrombotic risk in technical aspects of different DAPT duration

Figure 3

The Kaplan-Meier estimate curve of different DAPT duration 